\title{
Contribution of a High Dose of L-Ascorbic Acid to Carnitine Synthesis in Guinea Pigs Fed High-Fat Diets
}

\author{
Megumi Otsuka, ${ }^{*}$ Miyuki MatsuZawa, Tae Youl HA ${ }^{1}$ \\ and Nobuhiko AraKawa \\ Department of Nutrition \& Food Science, Ochanomizu University, \\ Bunkyo-ku, Tokyo 112-8610, Japan \\ ${ }^{1}$ Rice Utilization Research Center, Korea Food Research Institute, \\ Seognam-Si, Kyonggi-Do, 463-420, Korea
}

(Received October 7, 1998)

\begin{abstract}
Summary Ascorbate is a cofactor of two-enzyme hydroxylation in the pathway of carnitine biosynthesis. The purpose of this study was to investigate the contribution of ascorbate to endogenous carnitine in guinea pigs fed high-fat diets. The contents of carnitine in plasma, urine and tissues of guinea pigs supplemented with L-ascorbic acid were determined and compared with those supplemented with carnitine. Albino-Hartley guinea pigs were fed vitamin C-deficient diets containing lard throughout the experiment. They were administered orally with $5 \mathrm{mg} \mathrm{L}$-ascorbic acid/d/animal for $14 \mathrm{~d}$, and then divided into three groups and administered orally with the following supplements (/d/animal) for $14 \mathrm{~d}$; L $(5 \mathrm{mg}$ L-ascorbic acid), LASA (100 $\mathrm{mg}$ L-ascorbic acid), and LCAR (10 mg carnitine plus $5 \mathrm{mg}$ L-ascorbic acid). As a control, a normal group was fed vitamin C-deficient diets and administered orally with $5 \mathrm{mg}$ L-ascorbic acid/d/animal for $28 \mathrm{~d}$. The animals fed high-fat diets (L group) had higher free-carnitine contents in the muscle and urine than the normal group. The groups of LCAR and LASA had significantly higher contents of acid-soluble carnitine $(p<0.05)$ in plasma than the $\mathrm{L}$ group. Urinary excretion of carnitine in the LASA group was decreased to the same level as that in the normal group, although no significant difference between the groups of L and LCAR was observed. Moreover, the supplement of ascorbic acid, but not of carnitine, induced a significantly lower content of triacylglycerol in the plasma of the LASA group as compared to the $\mathrm{L}$ group $(p<0.05)$. These data suggest that high doses of ascorbic acid in guinea pigs fed high-fat diets contribute to the enhancement of carnitine synthesis and improvement of the triacylglycerol content in the plasma.
\end{abstract} Key Words L-ascorbic acid, carnitine, guinea pigs, plasma triacylglycerol, high-fat diets

*To whom correspondence should be addressed. E-mail: m220@cc.ocha.ac.jp 
Many studies have reported that carnitine can alter the lipid metabolism in patients with hyperlipidemia and atherosclerosis, and in parenteral nutrition (1-7), modify the physical performance $(8,9)$ and, moreover, delay fatigue $(10,11)$. Cederblad (12) reported that high-fat diets caused a significant increase of acyl carnitine in rat plasma and a higher clearance of carnitine than low-fat diets. It is assumed that the lowering effect of exogenous carnitine on plasma triacylglycerol in fat-loaded rats is based on the enhancement of $\beta$-oxidation of fatty acids (13).

Ascorbate is known to be a cofactor in two-enzyme hydroxylation in the pathway of carnitine biosynthesis. We found that ascorbate enhanced carnitine synthesis, which in turn stimulated the $\beta$-oxidation of fatty acids using primary cultured guinea pig hepatocytes $(14,15)$. Furthermore, we observed that vitamin $\mathrm{C}$ deficiency led to a decrease of carnitine content in tissues and an increase of triacylglycerol content in the plasma of guinea pigs (16). Considering the report showing that the decrease of carnitine level in the tissue of scorbutic guinea pigs was due to the decrease of its renal reabsorption, but not of its synthesis (17), the low content of ascorbate in the liver and kidney of scorbutic animals is likely to be sufficient for hydroxylation in the pathway of carnitine synthesis. In contrast, we found that a high dose of ascorbic acid to guinea pigs had no effect on the increase of carnitine contents in tissues (14), however, it remains unclarified whether high doses of ascorbic acid have some advantage on the increase of endogenous carnitine on certain physical or clinical conditions required for higher amounts of carnitine. Although an improvement of lipid metabolism by a high dose of ascorbic acid has been reported $(14,18)$, there are few studies on the relation between endogenous carnitine and fatty acid oxidation in mammals.

The purpose of this study was to clarify the role of ascorbic acid on carnitine synthesis using guinea pigs, which cannot synthesize ascorbic acid, fed high-fat diets. The effects of a high dose of ascorbic acid on the accumulation of carnitine in tissues, its urinary excretion and plasma triacylglycerol content were examined and compared with those of exogenous carnitine.

\section{MATERIALS AND METHODS}

Materials. L-Carnitine was obtained from Sigma Chemical (St. Louis, MO, USA). L-Ascorbic acid was purchased from Wako Pure Chemical Ind. (Osaka, Japan). NAD, NADPH and carnitine acetyltransferase were obtained from Boehringer Mannheim GmbH (Germany).

Animals. Male albino guinea pigs (Hartley strain, $221 \pm 3 \mathrm{~g}$ mean \pm SE body weight) from Saitama Animal Research (Saitama, Japan) were housed individually in stainless steel cages at $22 \pm 2{ }^{\circ} \mathrm{C}$ and $55 \pm 3 \%$ relative humidity with a $12-\mathrm{h}$ light-dark cycle. The guinea pigs were maintained in accordance with the Guidelines for Animal Experimentation of Ochanomizu University. The animals were given free access to a commercial diet (CG-7, Clea Japan, Tokyo, Japan) and water for $4 \mathrm{~d}$.

Diets. All diets were based on vitamin C-deficient diets formulated by the 
manufacturer (Clea Japan) to be identical to the commercial diet, CG-7. The only modification was that no vitamin $\mathrm{C}$ was added. The composition of these custom-prepared basal diets ( $10 \%$ of the energy value of diet as fat) was as follows (g/kg diet): water, 88; crude protein, 207; crude fat, 40; fiber, 89; ash, 79; and energy, $13 \mathrm{~kJ} / \mathrm{g}$ (manufacturer's analysis). Experimental diets containing lard (28\% of the energy value of the diet as fat) prepared by the addition of $6 \%$ lard to vitamin C-deficient diets were obtained from Clea Japan. Composition of the experimental diets was as follows ( $\mathrm{g} / \mathrm{kg}$ diet): water, 83; crude protein, 195; crude fat, 94; fiber, 84; ash, 74; and energy, $15 \mathrm{~kJ} / \mathrm{g}$. The carnitine content of the diets used in this study was under $0.2 \mu \mathrm{g} / \mathrm{kg}$ diet (estimated intake under $10 \mathrm{ng} / \mathrm{animal} / \mathrm{d}$ ) and exogenous carnitine was negligible. All diets were provided in pellet form.

Experimental design. Twenty animals were divided into two dietary groups after acclimatization. Five animals in the normal group were fed vitamin C-deficient diets and administered orally with $5 \mathrm{mg}$ L-ascorbic acid per day per animal for $28 \mathrm{~d}$. The other animals were fed experimental diets containing lard throughout the experimental period and administered orally with $5 \mathrm{mg} \mathrm{L}$-ascorbic acid for $14 \mathrm{~d}$. On day 15 , they were divided into three groups ( $n=5$ per group) and administered orally with $5 \mathrm{mg} \mathrm{L}$-ascorbic acid (L), $100 \mathrm{mg} \mathrm{L}$-ascorbic acid (LASA), or $10 \mathrm{mg}$ carnitine plus $5 \mathrm{mg}$ L-ascorbic acid per day per animal (LCAR) for $14 \mathrm{~d}$. A supplement of $5 \mathrm{mg}$ ascorbic acid for the groups of L and LCAR was chosen in order to prevent scurvy and maintain health, while a supplement of $10 \mathrm{mg}$ carnitine for the LCAR group was selected in order to avoid tissue saturation and excess amounts of urinary excretion of carnitine, which was based on our preliminary data about the tissue distribution and urinary excretion by graded doses of carnitine in guinea pigs. All animals were given the respective supplement dissolved in $0.5 \mathrm{~mL}$ of saline immediately before use. Body weight was measured twice weekly and food intake was determined daily. During the last week of feeding (days 23 to 28), urine was collected into a flask with $0.45 \mathrm{~mol} / \mathrm{L}$ perchloric acid for $24 \mathrm{~h}$, and stored at $-30^{\circ} \mathrm{C}$. At the end of the experiment, guinea pigs were anesthetized intraperitoneally with pentobarbital and blood was obtained via the aorta vessel. After removing blood, the liver, heart and muscle from a hind leg were elicited, weighed, and stored at $-80^{\circ} \mathrm{C}$ for subsequent analysis.

Measurements and analytical procedures. The carnitine in the plasma and tissues was extracted with $0.45 \mathrm{~mol} / \mathrm{L}$ perchloric acid. After centrifugation of the perchloric acid extracts including urine, the supernatant was stored at $-80^{\circ} \mathrm{C}$ until analysis. The contents of acid-soluble and free carnitine in the supernatants were determined by the HPLC method described in Arakawa et al (19). The content of each carnitine in urine was corrected by urinary creatinine concentration determined by the Wako Creatinine-Test (Wako Pure Chemical Ind.). Triacylglycerol in the plasma was determined using the Triglyceride E-Test (Wako Pure Chemical. Ind.).

Statistical analysis. Results are expressed as means with their standard errors. Statistical significance within a group was determined by one-way ANOVA followed, where appropriate, by Fisher's test of least difference for multiple comparisons to 

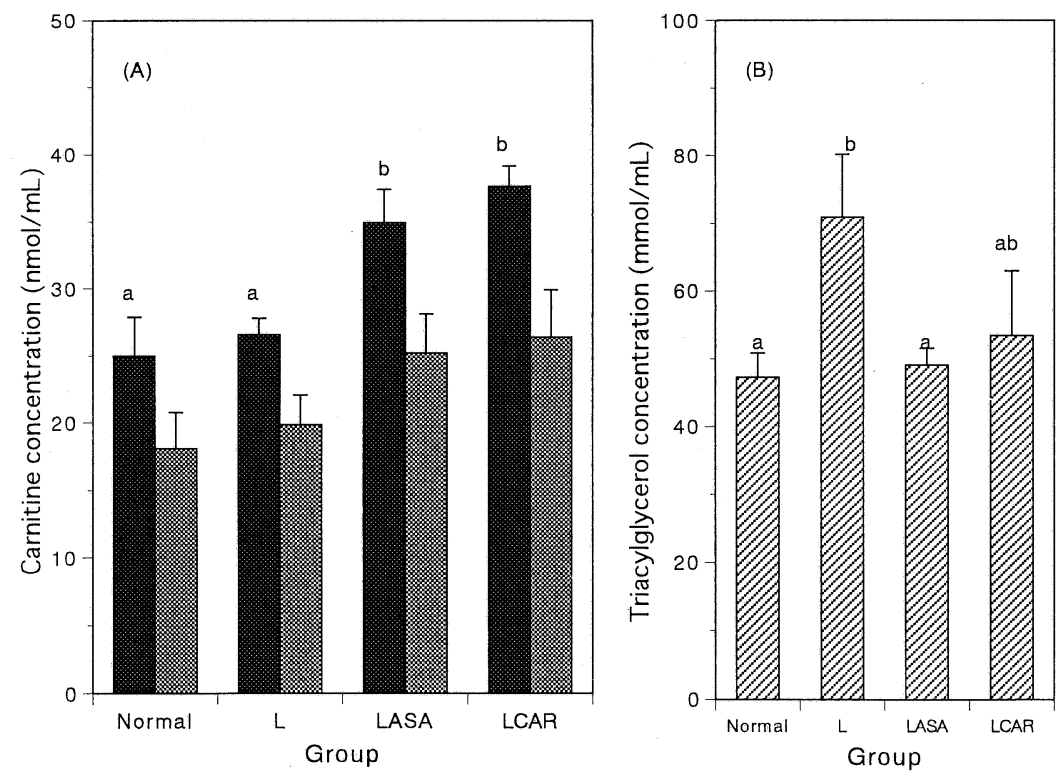

Fig. 1. The contents of carnitine (A) and triacylglycerol (B) in the plasma of guinea pigs fed normal or high-fat diets. Means \pm SE ( $n=4$ or 5 ). Bars in A represent acid-soluble carnitine (black bar) and free carnitine (gray bar). Bars with different letters were significantly different $(p<0.05)$. The normal group was fed ascorbatedeficient diets and supplemented orally with $5 \mathrm{mg}$ per day per animal for $28 \mathrm{~d}$. The guinea pigs in groups L, LASA and LCAR were fed vitamin C-deficient diets containing lard throughout the experiment and administered orally the following supplements for the latter half: L, $5 \mathrm{mg}$ ascorbate; LASA, $100 \mathrm{mg}$ ascorbate; LCAR, $10 \mathrm{mg}$ carnitine plus $5 \mathrm{mg}$ ascorbate.

compare the means between groups using Statview Software, Version 4.51, 1996 (Abacus Concepts, Berkeley, CA, USA). A $p$ value of $<0.05$ was statistically significant.

\section{RESULTS}

The final body weight of the L group $(432 \pm 25 \mathrm{~g}$, mean \pm SE) was significantly higher than that of the normal group $(359 \pm 24 \mathrm{~g}, p<0.05)$, while it was not significantly different from that of the LASA and LCAR groups $(429 \pm 13 \mathrm{~g}$ and $393 \pm 27 \mathrm{~g}$, respectively) since their food consumption matched that of the L group in order to have equal energy. No significant difference in tissue weight was observed among the groups.

Figure 1A illustrates the concentrations of acid-soluble and free carnitine in the plasma. The diets containing lard had no effect on the concentration of either carnitine, however, the groups of LASA and LCAR had significantly higher con- 
Table 1. Carnitine concentration in the livers of guinea pigs fed high-fat diets.

\begin{tabular}{lccc}
\hline \multirow{2}{*}{ Group (n) } & \multicolumn{3}{c}{ Carnitine concentration (nmol/g tissue) } \\
\cline { 2 - 4 } & Acid-soluble & Free & Esterified \\
\hline Normal (4) & $278 \pm 47.9^{*}$ & $254 \pm 48.4$ & $24.5 \pm 2.0^{\mathrm{ab}}$ \\
L (4) & $246 \pm 33.5$ & $230 \pm 35.0$ & $14.8 \pm 2.1^{\mathrm{a}}$ \\
LASA (4) & $274 \pm 26.8$ & $245 \pm 20.1$ & $28.5 \pm 7.8^{\mathrm{b}}$ \\
LCAR (5) & $357 \pm 47.4$ & $345 \pm 48.4$ & $12.1 \pm 2.4^{\mathrm{a}}$ \\
\hline
\end{tabular}

* Mean \pm SE. ${ }^{\text {ab }}$ Significant difference with different letters in the same column $(p<$ $0.05)$.

Groups are the same as in Fig. 1. Esterified carnitine was calculated by the subtraction of free carnitine from acid-soluble carnitine.

Table 2. Carnitine concentration in the muscles of guinea pigs fed high-fat diets.

\begin{tabular}{lccc}
\hline \multirow{2}{*}{ Group (n) } & \multicolumn{3}{c}{ Carnitine concentration (nmol/g tissue) } \\
\cline { 2 - 4 } & Acid-soluble & Free & Esterified \\
\hline Normal (4) & $348 \pm 24.5$ & $251 \pm 22.2^{\mathrm{a}}$ & $96.2 \pm 13.0^{\mathrm{c}}$ \\
L (4) & $398 \pm 28.6$ & $345 \pm 30.6^{\mathrm{b}}$ & $52.7 \pm 5.9^{\mathrm{ab}}$ \\
LASA (4) & $361 \pm 34.4$ & $316 \pm 25.3^{\mathrm{b}}$ & $44.8 \pm 10.2^{\mathrm{a}}$ \\
LCAR (5) & $407 \pm 5.4$ & $333 \pm 3.7^{\mathrm{b}}$ & $73.9 \pm 3.7^{\mathrm{bc}}$ \\
\hline
\end{tabular}
$0.05)$.

* Mean \pm SE. ${ }^{\text {abc }}$ Significant difference with different letters in the same column $(p<$

Groups are the same as in Fig. 1. Esterified carnitine was calculated by the subtraction of free carnitine from acid-soluble carnitine.

centrations of acid-soluble carnitine than $\mathrm{L}$ group $(p<0.05)$, indicating that the supplement of $100 \mathrm{mg}$ ascorbic acid or $10 \mathrm{mg}$ carnitine was effective to increase the carnitine in plasma. No significant difference in the concentrations of free carnitine, and also esterified carnitine (data not shown), which was obtained by subtraction of the free-carnitine concentration from acid-soluble carnitine, was observed among the groups. Figure 1B shows the concentration of triacylglycerol in the plasma. The L group was significantly higher than the normal group $(p<0.05)$, whereas the LASA group, but not the LCAR group, was significantly lower than the L group $(p<0.05)$.

Table 1 shows the concentration of carnitine in the liver. There was no statistical significance of acid-soluble or free-carnitine concentrations among the groups, however, the concentration of esterified carnitine in the LASA group was significantly higher than that in the L group.

In muscle, the groups fed a high-fat diet had significantly higher concentrations of free carnitine than the normal group $(p<0.05)$ as shown in Table 2 . In contrast, 


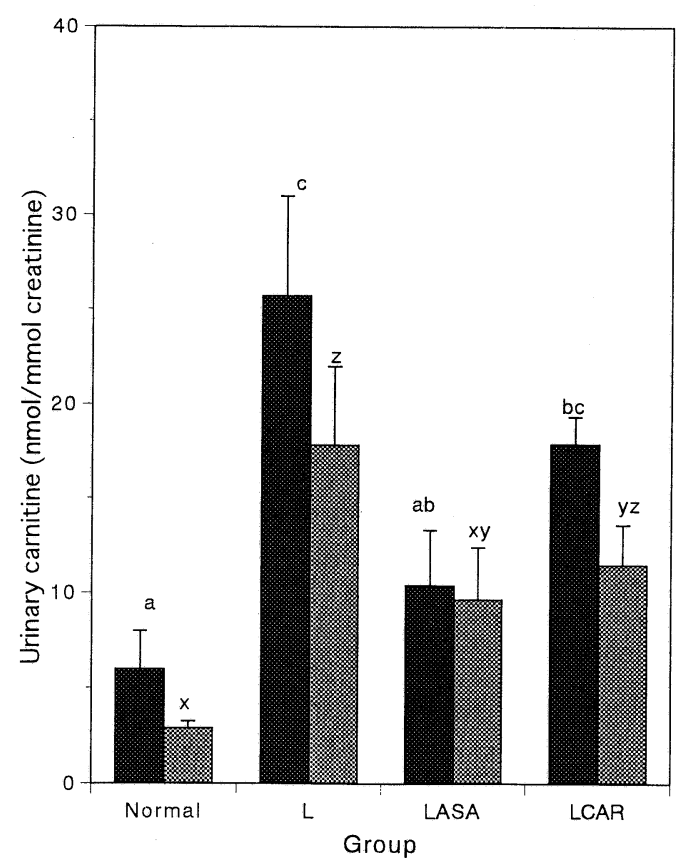

Fig. 2. Urinary excretion of the carnitine contents in guinea pigs fed normal or high-fat diets. Groups and symbols are the same as those in Fig. 1. Different letters in each bar indicate significantly different results $(p<0.05)$.

the concentration of esterified carnitine in the L group was significantly lower than that in the normal group $(p<0.05)$; that is, high-fat diets resulted in the increase of free carnitine and clearance of esterified carnitine. There was no significant effect of the supplement of ascorbic acid or carnitine on the concentrations of carnitine as compared with the L group.

Urinary excretion is shown in Fig. 2. High-fat diets induced significantly higher excretion of acid-soluble and free carnitine than the normal group $(p<0.05)$, although no statistical significance in esterified carnitine was observed (data not shown). The urinary excretion of acid-soluble and free carnitine in the LASA group, but not in the LCAR group, was significantly lower than in the L group $(p<0.05)$, and it was decreased to the same level as in the normal group.

\section{DISCUSSION}

Several studies have demonstrated the effects of the diet composition on carnitine distribution $(7,20)$. For instance, the urinary excretion of free and esterified carnitine in humans was increased by high-fat diets (12). In this study using guinea pigs fed high-fat diets, the urinary excretion of free carnitine was increased. Although the tissue distribution of carnitine differs with the species and feeding period, our 
result for guinea pigs is consistent with that for humans. Cederblad (12) has suggested that an increase of urinary carnitine excretion by specimens on high-fat diets is due to an increase of dietary carnitine, enhancement of carnitine synthesis, or the redistribution of tissue carnitine. Exogenous carnitine was negligible in the diets used in this study. Therefore, the increasing urinary excretion of carnitine may be due to either the enhancement of carnitine synthesis or the redistribution of tissue carnitine. Based on our data indicating that the free-carnitine concentration in the muscle of guinea pigs fed high-fat diets was increased, it is supposed that urinary excretion may reflect enhancement of the synthesis.

Ruff et al (21) reported that, in rats on a normal diet, approximately $60 \%$ of the carnitine administered at a dose above $1.3 \mathrm{mg} / 100 \mathrm{~g}$ body weight was recovered in the urine within $24 \mathrm{~h}$. The synthesis of endogenous carnitine is presumably lowered by supplementing with carnitine although the reducing level is dependent on its dose. In our preliminary experiment, the urinary excretion of acid-soluble carnitine for normal diets with $10 \mathrm{mg}$ carnitine was $35 \mathrm{nmol} / \mathrm{mmol}$ creatinine, which was twice as much as that for high-fat diets (Fig. 2). This indicates that the supplement of carnitine in high-fat diets may suppress urinary loss of carnitine since it is required for enhancing the $\beta$-oxidation of fatty acids in tissues.

The effects of a high dose of ascorbic acid on guinea pigs fed high-fat diets were as follows: 1) An increase of acid-soluble carnitine in the plasma, at least to the same extent as the supplement of $10 \mathrm{mg}$ carnitine; 2) an increase of esterified carnitine in the liver; 3) a decrease of esterified carnitine in the urine; and 4) a decrease of plasma triacylglycerol. From these data, we hypothesize that the synthesis of endogenous carnitine in the liver is stimulated by ascorbate, which in turn, increases the concentration of carnitine in the plasma, while the increase of endogenous carnitine resulted in the enhancement of fatty acid transport into mitochondria in the liver, followed by the acceleration of $\beta$-oxidation of fatty acids and an increase in esterified carnitine.

Using primary cultured hepatocytes $(16,22)$, we found that loading with fatty acids did not affect the uptake of free carnitine, but increased cellular esterified carnitine. In addition, the supplement of carnitine or ascorbate with the precursor in the presence of fatty acids induced a decrease in cellular triacylglycerol and increase in $\beta$-hydroxybutyrate secretion; that is, the enhancement of $\beta$-oxidation. In this study, the supplement of ascorbic acid, but not of carnitine, caused a decrease in triacylglycerol concentration although both supplements attributed to the increase of carnitine concentrations in the plasma. Thus, a high dose of ascorbic acid may have advantages over the improvement of plasma triacylglycerol concentration as compared with exogenous carnitine because of the limitation on uptake of carnitine into the liver by a single oral dose. In other words, this indicates that endogenous carnitine is more effective than exogenous carnitine for the transport of fatty acids into mitochondria in the liver and the reduction of triacylglycerol in the plasma. The data for higher esterified carnitine in the liver with the supplement of ascorbic acid reveal that endogenous carnitine stimulates the $\beta$-oxidation of fatty acids, 
although the contents of total fatty acids in the liver were not significantly different among the groups within this experimental period (data not shown).

In muscle, which contains most of the carnitine in the body, we found higher esterified carnitine in the LCAR group than in the groups of L and LASA; therefore, exogenous carnitine may be easily transported into the muscle in order to trap acyl groups. Esterified carnitine may be an appropriate form to use acyl groups for energy metabolism in tissues, since it can be freely transported within and outside the membrane of mitochondria. These data may also support the hypothesis by Harris et al (23) suggesting that carnitine serves as a buffer for the metabolically important acetyl-CoA pool in tissues.

Englard and Carnicero (24) demonstrated that the activity of $\gamma$-butyrobetaine hydroxylase in guinea pig liver was five times higher than in rat liver. Consequently, carnitine synthesis in guinea pigs, especially on high-fat diets, may be regulated more sensitively by the level of L-ascorbic acid in order to sustain the activity (25). In addition, the evidence of improvement in plasma triacylglycerol suggests that higher amounts of ascorbic acid can contribute to modification of the lipid metabolism in plasma. It is difficult to refer adequate amounts of ascorbic acid in order to enhance the synthesis of endogenous carnitine, however, saturation of the body pool is presumably useful for the stimulation of hydroxylase activity by considering the data that the concentration of ascorbic acid in the liver of the LASA group was about three times higher than that of the $\mathrm{L}$ group (data not shown).

In conclusion, it is assumed that high doses of ascorbic acid in guinea pigs fed high-fat diets contribute to an increase in endogenous carnitine and improvement of the triacylglycerol concentration in the plasma.

\section{REFERENCES}

1) Maebashi M, Kawamura N, Sato M, Imamura A, Yoshinaga K. 1978. Lipid-lowering effect of carnitine in patients with type-IV hyperlipoproteinemia. Lancet 2: 805-807.

2) Orzali A, Maetzke G, Donzelli F, Rubaltelli FF. 1984. Effect of carnitine on lipid metabolism in the neonate. II. Carnitine addition to lipid infusion during prolonged total parenteral nutrition. $J$ Pediatr 104: 436-440.

3) Davis AT, Albrecht RM, Scholten DJ, Morgan RE. 1988. Increased plasma carnitine in trauma patients given lipid-supplemented total parenteral nutrition. Am J Clin Nutr 8: $1400-1402$.

4) Olson AL, Nelson SE, Rebouche CJ. 1989. Low carnitine intake and altered lipid metabolism in infants. Am J Clin Nutr 49: 624-628.

5) Rebouche CJ, Panagides DD, Nelson SE. 1990. Role of carnitine in utilization of dietary medium-chain triglycerides by term infants. Am J Clin Nutr 52: 820-824.

6) Wanner C, Wackerle B, Boeckle H, Schollmeyer P, Horl WH. 1990. Plasma and red blood cell carnitine and carnitine esters during L-carnitine therapy in hemodialysis patients. Am J Clin Nutr 51: 407-410.

7) Dayanandan A, Kumar P, Kalaiselvi T, Panneerselvam C. 1994. Effect of L-carnitine on blood lipid composition in atherosclerotic rats. J Clin Biochem Nutr 17: 81-87.

8) Hiatt WR, Nawaz D, Brass EP. 1987. Carnitine metabolism during exercise in patients 
with peripheral vascular disease. J Appl Physiol 62: 2383-2387.

9) Vukovich MD, Costill DL, Fink WJ. 1994. Carnitine supplementation: effect on muscle carnitine and glycogen content during exercise. Med Sci Sports Exerc 26: 1122-1129.

10) Brass EP, Scarrow AM, Ruff LJ, Masterson KA, Lunteren EV. 1993. Carnitine delays rat skeletal muscle fatigue in vitro. J Appl Physiol 75: 1595-1600.

11) Kuratsune H, Yamaguti K, Takahashi M, Tagawa S, Machii T, Kitani T. 1995. Abnormal cellular carnitine metabolism in chronic fatigue syndrome. $J$ Immunol Immunopharmacol 15: 40-44.

12) Cederblad G. 1987. Effect of diet on plasma carnitine levels and urinary carnitine excretion in humans. Am J Clin Nutr 45: 725-729.

13) Maccari F, Arseni A, Chiodi P, Ramacci MT, Angelucci L, Hulsmann WC. 1987. L-Carnitine effect on plasma lipoproteins of hyperlipidemic fat-loaded rats. Lipids 22: $1005-1008$.

14) Ha T-Y, Otsuka M, Arakawa N. 1991. The regulatory effect of L-ascorbic acid on the carnitine synthesis in primary cultured guinea pigs hepatocytes. J Nutr Sci Vitaminol 37: 371-378.

15) Ha T-Y, Otsuka M, Arakawa N. 1994. L-Ascorbic acid indirectly stimulates fatty acid utilization in primary cultured guinea pigs hepatocytes by enhancing carnitine synthesis. $J$ Nutr 124: 732-737.

16) Ha T-Y, Otsuka M, Arakawa N. 1990. The effect of graded doses of ascorbic acid on the tissue carnitine and plasma lipid concentration. J Nutr Sci Vitaminol 36: 227-234.

17) Rebouche CJ. 1995. Renal handling of carnitine in experimental vitamin C deficiency. Metabolism 44: 1639-1643.

18) Bobek P, Ginter E, Ozdin L, Poledne R, Potucek J. 1983. Effect of long-term marginal vitamin $\mathrm{C}$ deficiency on plasma triglyceride kinetics in guinea-pigs. Biomed Biochim Acta 42: 413-416.

19) Arakawa N, Ha T-Y, Otsuka M. 1989. An improved high-performance liquid chromatographic assay for the determination of free and esterified carnitine in animal tissues. J Nutr Sci Vitaminol 35: 475-479.

20) Dtadler DD, Chenard CA, Rebouche CJ. 1993. Effect of dietary macronutrient content on carnitine excretion and efficiency of carnitine reabsorption. Am J Clin Nutr 58: 868-872

21) Ruff LJ, Miller LG, Brass EP. 1991. Effect of exogenous carnitine on carnitine homeostasis in the rat. Biochim Biophys Acta 1073: 543-549.

22) Ha T-Y, Otsuka M, Arakawa N. 1993. The behavior of carnitine in primary-cultured guinea pig hepatocytes. Nippon Eiyo Shokuryo Gakkaishi (J Jpn Soc Nutr Food Sci) 46: $483-486$.

23) Harris RC, Foster CV, Hultman E. 1987. Acetylcarnitine formation during intense muscular contraction in humans. J Appl Physiol 63: 440-442.

24) Englard S, Carnicero HH. 1987. $\gamma$-Butyrobetaine hydroxylation to carnitine in mammalian kidney. Arch Biochem Biophys 190: 361-364.

25) Rebouche CJ. 1991. Ascorbic acid and carnitine biosynthesis. Am J Clin Nutr 54: $1147 \mathrm{~S}-1152 \mathrm{~S}$. 